\title{
Edge Detection (Image Processing) Smart Traffic Control
}

\author{
Brinda.R. B ${ }^{1}$, Namratha Venkatesh Murthy ${ }^{2}$, B. M. Ramya ${ }^{3}$, Faculty Advisor: Dr. Vijaya Prakash A M \\ Bangalore Institute of Technology 1,2,3,4
}

\begin{abstract}
The frequent traffic jams at major junctions call for an efficient traffic management system in place $[1,2]$.The resulting wastage of time and increase in pollution levels can be eliminated on a city-wide scale by these systems [2]. The paper proposes to implement a smart traffic controller using real time image processing. The image sequences from a camera are analyzed using various edge detection Algorithms and object counting methods. Subsequently, the number of vehicles at the intersection is evaluated and traffic is efficiently managed [7]. The paper also proposes to implement a real- time emergency vehicle detection system. In case an emergency vehicle is detected, the lane is given priority over all the others [5].
\end{abstract}

Keywords: Image Processing, Smart Traffic Control, Edge Detection, Real- Time Emergency.

\section{INTRODUCTION}

This paper will be mainly focused on reducing and controlling the various traffic problems faced by cities today. Current traffic control techniques using magnetic loops, infra red systems and inductive loops are subject to a high failure rate when installed in poor road surfaces and require separate systems for traffic counting and for traffic surveillance. However, by using video based systems, they provide more traffic information, combine both surveillance and traffic control technologies, are easily installed, and are scalable with progress in image processing techniques.

Edge detection is one of the most commonly used operations in image analysis. The reason for this is that edges form the outline

The image sequences from a camera are analyzed using various edge detection and object counting methods to obtain the most efficient algorithm. Subsequently, the number of vehicles at the intersection is evaluated and traffic is efficiently managed. Also, if an emergency vehicle is detected, it may be directed towards the least congested lane which will be given a higher priority compared to the others.

Video motion detection is fundamental in many autonomous video surveillance strategies. However, in outdoor scenes inconsistent lighting and background movement is a challenging problem. Light may change anytime which cause system output less meaningful to deal with. Recent research has produced several background modeling techniques based on image differencing, that exhibit real-time performance and high accuracy for certain classes of scenes where the weather introduces unpredictable variations in both lighting and background movement.

\section{II.METHODOLOGY}

Identifying moving objects from a video sequence is a fundamental and critical task in video surveillance, traffic monitoring and analysis. A common approach in identifying the moving objects is background subtraction, where each video frame is compared against a reference or background model. Pixels in the current frame that deviate significantly from the background are considered to be moving objects.

These "foreground" pixels are further processed for object localization and tracking. Since background subtraction is often the first step in many computer vision applications, it is important that the extracted foreground pixels accurately correspond to the moving objects of interest. Moving object detection is the basic step for further analysis of video. It handles segmentation of moving objects from stationary background objects. This not only creates a focus of attention for higher level processing but also decreases computation time considerably. The various steps of our proposed system are a camera (webcam) is fixed on polls or other tall structures to overlook the traffic. Each frame extracted from the video are then analyzed to track, detect and count vehicles as seen in [9]. Then depending on the signal cycle ( 1 minute), time is allotted to each lane. For example, if the number of vehicles in a four- lane intersection is found to be 10,30 , 20 and 5 , then signal are changed depending upon the count of vehicles. The lane which contains 30 vehicles is given green signal first and then the lane with 20 and then lane with 10 and lane with 5 vehicles.

The following steps are performed for the above mentioned objective:

Step1: Webcam initialization is done by properly selecting adaptor name, device name and format.

Step2: Delete the previous video input object and all the video source objects associated with the previous video input object.

Step3: Capture sequences of video frames by specifying the number of frames required.

Step4: Perform the background modeling in the video.

Step5: Perform the background subtraction.

Step6: Detect the moving vehicle.

Step7: Perform the tracking of moving object

Step8: Perform the noise removal by properly selecting the filters.

Step9: Perform the counting of moving vehicle. 
Step10: Perform the traffic signal control depending count of vehicles using counter.

Step 11:If emergency vehicle detected, prioritize the lane Step12: Perform the background updating.

\section{III.PROPOSED SYSTEM}

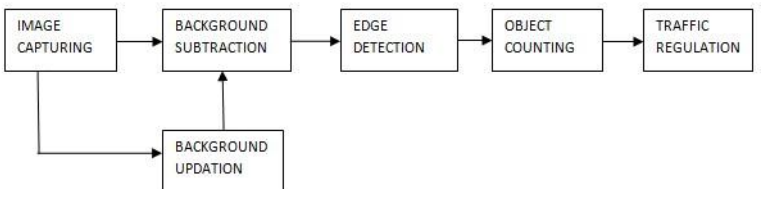

Figure 1: Block Diagram of proposed system

The various steps of our proposed system are described in Figure 1. A camera is fixed on poles or other tall structures to overlook the traffic scene as seen in [3]. Images extracted from the video are then analyzed to detect and count vehicles. Then depending on the signal- cycle (we have taken it to be 1 minute), time is allotted to each lane. For example, if the number of vehicles in a four-lane intersection is found to be $10,30,20$ and 20 , then time allotted to each lane is in the ratio $1: 3: 2: 2$.

The system also takes into account the emergency vehicles at the intersection. If such a vehicle is detected, the lane is given priority over the others

\section{A. BACKGROUND SUBTRACTION}

Background subtraction is a computational vision process of extracting foreground objects in a particular scene. A foreground object can be described as an object of attention which helps in reducing the amount of data to be processed as well as provide important information to the task under consideration. Often, the foreground object can be thought of as a coherently moving object in a scene. We must emphasize the word coherent here because if a person is walking in front of moving leaves, the person forms the foreground object while leaves though having motion associated with them are considered background due to its repetitive behavior. In some cases, distance of the moving object also forms a basis for it to be considered a background, e.g. if in a scene one person is close to the camera while there is a person far away in background, in this case the nearby person is considered as foreground while the person far away is ignored due to its small size and the lack of information that it provides. Identifying moving objects from a video sequence is a fundamental and critical task in many computer-vision applications.

Generating the current background image based on segmentation results extracted from differencing the image with the previous extracted background is the basic idea of our method. The updated background (Bnew) is computed as a function of current background (Bo) and current frame I through the equation as seen in [4]:

Where RES is the result of subtraction of consecutive frames, and the value of $\alpha$ is 0.5 .

$$
\text { Bnew }= \begin{cases}\left(\alpha * B_{0}\right)+(1-\alpha) * I_{s} \text { if RES }=0 \\ B_{0 *} \text { OTHERWSE }\end{cases}
$$

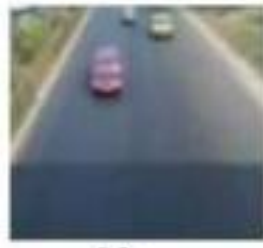

(a)

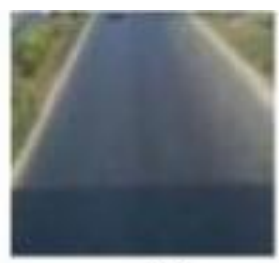

(b)

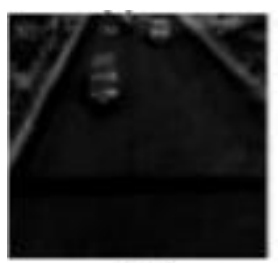

(c)

Figure 2: (a) Real-time image (b) Background image (c) Subtracted image

Identifying moving objects from a video sequence is a fundamental and critical task in many computer-vision applications. The following processes are employed

1. Image acquisition.

2. RGB to gray conversion.

3. Image enhancement.

4. Image matching using edge detection.

1. Image Acquisition: Initially image acquisition is done with the help of web camera. First image of the road is captured, when there is no traffic on the road. This empty road's image is saved as reference image at a particular site specified in the program

2. RGB to Gray Conversion: RGB to gray conversion is done on the progression of captured images. Now gamma correction is done on each of the captured gray image to achieve image enhancement.

3. Image Enhancement: The acquired image in RGB is first converted into gray. Now we want to bring our image in contrast to background so that the appropriate threshold level may be selected while binary conversion is carried out. This calls for image enhancement techniques. The objective of enhancement is to process an image so that result is more suitable than the original image for the specific application.

4. Image Matching Using Edge Detection: Edge detection methods locate the pixels in the image that correspond to the edges.

\section{IV.EDGE DETECTION}

Edge detection is one of the most commonly used operations in image analysis. The reason for this is that edges form the outline of an object. An edge is the boundary between an object and the background, and indicates the boundary between overlapping objects. This means that if the edges in an image can be identified accurately, all of the objects can be located and basic properties such as area, perimeter, and shape can be measured. Since computer vision involves the identification and classification of objects in an image, edge detections is an essential tool.

Upon comparison of various edge detection algorithms, it 
was inferred that Canny Edge Detector technique is the most efficient one as seen in [8]. Hence we are employing the Canny Edge Detector technique for our proposed model.

A. The Canny Edge Detector: The Canny edge detector is widely considered to be the standard edge detection algorithm in the industry. The steps in the Canny Edge Detector are as follows:

1. Smooth the image with a two dimensional Gaussian. In most cases the computation of a two dimensional Gaussian is costly, so it is approximated by two one dimensional Gaussians, one in the $\mathrm{x}$ direction and the other in the $\mathrm{y}$ direction.

2. Take the gradient of the image. This shows changes in intensity, which indicates the presence of edges. This actually gives two results, the gradient in the $\mathrm{x}$ direction and the gradient in the y direction.

3. Non-maximal suppression. Edges will occur at points the where the gradient is at a maximum. Therefore, all points not at a maximum should be suppressed. In order to do this, the magnitude and direction of the gradient is computed at each pixel. Then for each pixel check if the magnitude of the gradient is greater at one pixel's distance away in either the positive or the negative direction perpendicular to the gradient. If the pixel is not greater than both, suppress it.

4. Edge Thresholding. The method of thresholding used by the Canny Edge Detector is referred to as "hysteresis". It makes use of both a high threshold and a low threshold. If a pixel has a value above the high threshold, it is set as an edge pixel. If a pixel has a value above the low threshold and is the neighbor of an edge pixel, it is set as an edge pixel as well. If a pixel has a value above the low threshold but is not the neighbor of an edge pixel, it is not set as an edge pixel. If a pixel has a value below the low threshold, it is never set as an edge pixel.

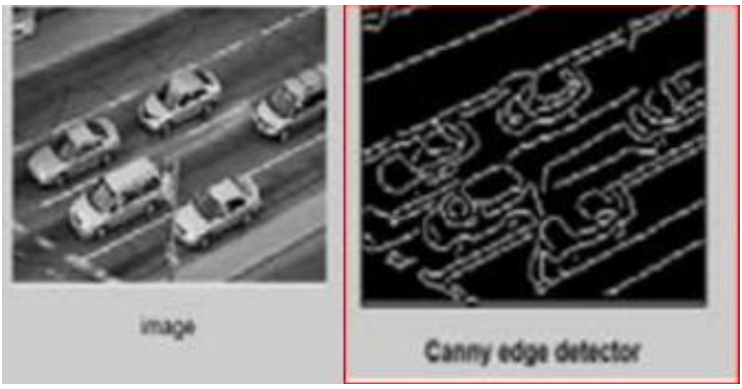

Figure 3: Output of canny edge detector

\section{OBJECT COUNTING}

Vehicle counting in traffic videos plays an essential role in traffic surveillance system. It can provide some important traffic information such as congestion level and statistical analysis of traffic flow. In this paper, a novel vehicle counting strategy, which is based on foreground detection algorithm in videos, is proposed as seen in [6]. Two advantages are contributed by our method. First, it is costsaving as no additional hardware devices are required. Second, the influence to performance caused by weather variations is eliminated by the use of foreground detection technique.

\section{EMERGENCY VEHICLE CONDITION}

The various steps of detection system are in Figure 5.In case of normal regulation the image is subjected to adaptive background subtraction, edge detection, and object counting methods. Using this, the number of vehicles is calculated and traffic is regulated as per the congestion in each lane.

In case a red beacon is detected, the next step is to identify whether it is from an emergency vehicle or not. This is done by identifying the blinking frequency of red light detected in the image sequence and comparing it to the standard used by the emergency vehicles as seen in [2].We scan the intermediate frames for the presence of the beacon. This is done by identifying the blinking frequency of red light detected in the image sequence and comparing it to the standard used by the emergency vehicles. We scan the intermediate frames for the presence of the beacon.

The conditions of detection of a red light beacon during various periods of the day are shown below. Night time conditions:

For red light beacon: $\mathrm{R}>230, \mathrm{G}<250, \mathrm{~B}<250$

In the intermediate frames: $\mathrm{R}<230, \mathrm{G}>230, \mathrm{~B}<230$.

Day time conditions:

For red light beacon: $\mathrm{R}>230, \mathrm{G}<250, \mathrm{~B}<250$

In the intermediate frames: $R<230, G<230, B<230$.

If matched the normal system is overridden and the lane is given priority over all the others. The lane is turned green until the vehicle has passed the intersection.

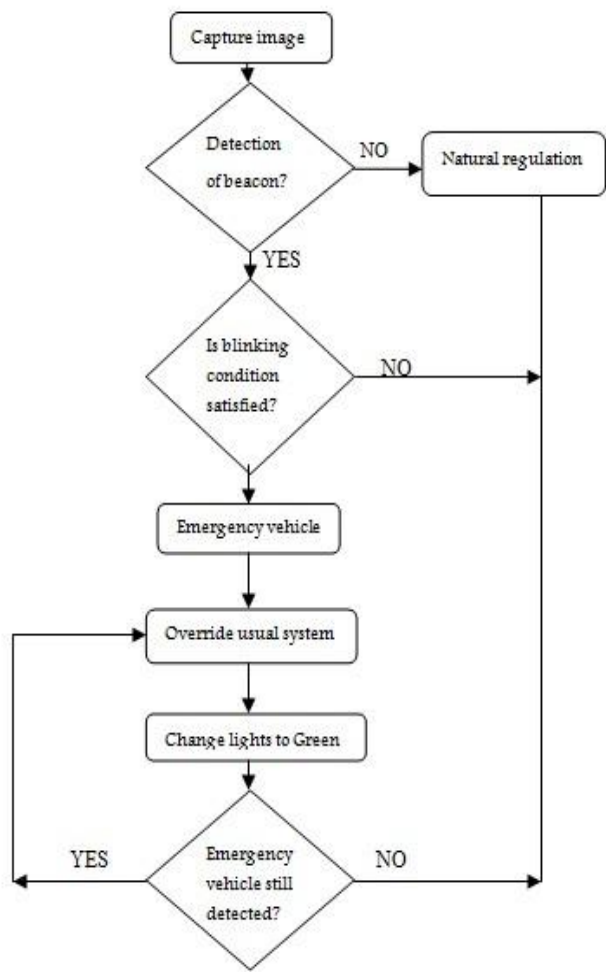

Figure 5: Flowchart for emergency vehicle detection

\section{CONCLUSION}

In this project, we have successfully implemented algorithm for a real-time image processing based smart traffic controller. It was inferred that Canny Edge Detector 
technique is the most efficient one. This paper demonstrates that image processing is a far more efficient method of traffic control as compared to traditional techniques. We have also implemented a system for emergency vehicle detection based on image processing techniques. The use of our algorithm removes the need for un-necessary hardware such as sound sensors or RFID tags. The increased response time for these vehicles is crucial for the prevention of loss of life.

\section{REFERENCES}

[1] Ahmed S. Salama, Bahaa K. Saleh, Mohamad M.Eassa"Intelligent Cross Road Traffic Management System (ICRTMS)"2nd Int. Conf .on Computer Technology and Development, Cairo, Nov 2010, pp. 27-31.

[2] Vikramaditya Dangi, Amol Parab, Kshitij Pawar, S.S Rathod, "Image Processing Based Intelligent Traffic Controller".

[3] Y. Wu, F. Lian, and T. Chang, "Traffic monitoring and vehicle tracking using roadside camera," IEEE Int. Conf. On Robotics and Automation, Taipei, Oct 2006, pp. 4631-4636.

[4] Jinglei, L. Zhengguang, and T. Univ, "A vision-based road surveillance system using improved background subtraction and region growing approach," Eighth ACIS Int. Conf. On Software Engineering, Artificial Intelligence, Networking, and Parallel/Distributed Computing, Qingdao, August 2007, pp. 819822.

[5] M. Siyal, and J. Ahmed,"A novel morphological edge detection and window based approach for real-time road data control and management," Fifth IEEE Int. Conference Information, Communications and signal Processing, Bangkok, July 2005, pp. 324-328.

[6] K. Wang, Z. Li, Q. Yao, W. Huang, and F. Wang, "An automated vehicle counting system for traffic surveillance," IEEE Int. Conference. On Vehicular Electronics and Safety, Japan, Dec 2007, pp. 1-6.

[7] M. Juneja, P. Sandhu, "Performance evaluation of edge detection techniques for images in spatial domain," Int. Journal of Computer Theory and Engineering, Singapore, vol. 1, no. 5, Dec 2009, pp. 1793-8201.

[8] Ehsan Nadernejad, Sara Sharifzadeh, Hamid Hassanpour, "Edge Detection Techniques: Evaluation and Comparisons", Applied Mathematical Sciences, Vol.2, 2008

[9] Somashekhar .G.C, Sarala Shirabadagi, Ravindra S. Hegadi, "High Density Traffic Management using Image Background Subtraction Algorithm", International Journal of Computer Applications(09758887), Recent Advances in Information Technology,2014. 